\section{Risk factors for intestinal obstruction after Ladd procedure}

\author{
Tetsuya Mitsunaga, Takeshi Saito, Keita \\ Terui, Mitsuyuki Nakata, Sachie Ohno, \\ Naoko Mise, Satoru Oita, Hideo Yoshida \\ Department of Pediatric Surgery, \\ Graduate School of Medicine, Chiba \\ University, Japan
}

\begin{abstract}
Intestinal obstruction is a common complication after Ladd procedure. Ninety-three cases who had undergone the Ladd procedure between 1977 and 2013 treated at our own institution were retrospectively reviewed to identify the causes and risk factors for intestinal obstruction. The Ladd procedure has been performed without any intestinal fixing. Of the 87 cases who survived to discharge, intestinal obstruction was observed in 22 (25.3\%). Among the cases with intestinal obstruction, 13 (59.1\%) showed intestinal ischemia at the initial operation; this incidence was notably high, although it is low when only those cases with another concurrent surgical digestive disease are considered. All cases of intestinal obstruction were caused not by recurrent volvulus, but by adhesion between the intestine and the mesentery. Intestinal fixing is not required to prevent recurrent volvulus, but it is important to achieve adequate widening of the mesenteric base. The risk of intestinal obstruction after the Ladd procedure, on the other hand, is high. Moreover, patients with intestinal ischemia have an increased risk of intestinal obstruction.
\end{abstract}

\section{Introduction}

The standard treatment for intestinal malrotation is the Ladd procedure, first described by Ladd in $1936 .{ }^{1}$ The prognosis of intestinal malrotation is good if there is no midgut volvulus, intestinal necrosis, prematurity, or other abnormalities. ${ }^{2}$ According to the Japanese national aggregate of neonatal surgery, in 2008 the mortality rate was reported to be $2.4 \%$. On the other hand, intestinal obstruction is a common complication after the Ladd procedure. ${ }^{3}$ The details of these postoperative intestinal obstructions, however, have been unclear, with the exception of several reports concerning recurrent midgut volvulus. ${ }^{4,5}$ Since its establishment in 1977, our department has performed the Ladd procedure without any intes- tinal fixing. The cases treated at our institution were reviewed in order to analyze the cause of and the risk factors for postoperative intestinal obstruction.

\section{Materials and Methods}

The hospital and office records of 93 patients who had undergone the Ladd procedure at Chiba University Hospital (Japan) between 1977 and 2013 were retrospectively reviewed. No patient treated during this period was excluded. Data were collected on sex, age, clinical findings, operative procedure, postoperative course, follow-up period, and long-term complications. The type of intestinal malrotation was categorized according to the classification reported by Touloukian et al. ${ }^{6}$

The Ladd procedure consists of the following steps: detorsion of the volvulus, followed by division of the Ladd bands, widening of the mesenteric base, appendectomy, and replacement of the intestines without rotation in the abdominal cavity and without any intestinal fixing. Cases in which the intestinal tract showed a color change to dark red at the laparotomy with or without recovery through detorsion or warming were treated for intestinal ischemia.

A clinical diagnosis of intestinal obstruction was considered for patients complaining of abdominal pain requiring a hospital stay with the presence of multiple gas-fluid levels on plain abdominal X-ray. The cases in which postoperative intestinal obstruction occurred were compared with those in which it did not occur in a statistical analysis of clinical factors. Statistical analysis was performed using Student's t-test for means and the KaplanMeier technique with comparison by the logrank test for frequencies. Probability values of less than 0.05 were considered significant.

\section{Results}

The patients' characteristics are listed in Table 1. Twenty-eight cases (30.1\%) had associated digestive surgical diseases, such as congenital diaphragmatic hernia, duodenal atresia, small intestinal atresia/stenosis, gastroschisis, omphalocele, or anorectal malformation. Seventy cases (75.3\%) were corrected in the neonatal period, seven cases (7.5\%) in infancy, six (6.5\%) in early childhood, and ten $(10.8 \%)$ in the elementary school years. As for the classification of malrotation cases, $46.2 \%$ were of the nonrotation type, and $51.6 \%$ were of the incomplete rotation type. Two cases were of the reverse rotation type with a paraduodenal hernia, a subtype of incomplete
Correspondence: Tetsuya Mitsunaga, Department of Pediatric Surgery, Graduate School of Medicine, Chiba University, 1-8-1 Inohana, Chuo-ku, Chiba 260-8677, Japan.

Tel. +81.43.222.7171 - Fax: +81.43.226.2366.

E-mail: tetsuya@z.email.ne.jp

Key words: Malrotation; midgut volvulus; postoperative intestinal obstruction; adhesion.

Contributions: the authors contributed equally.

Conflict of interest: the authors declare no potential conflict of interest.

Conference presentation: part of this paper was presented at the 51st Annual Meeting of the Japanese Society of Pediatric Surgeons, 2014 May 8-10, Osaka, Japan.

Received for publication: 6 January 2015.

Revision received: 10 February 2015.

Accepted for publication: 10 February 2015.

This work is licensed under a Creative Commons Attribution NonCommercial 3.0 License (CC BYNC 3.0).

(C) Copyright T. Mitsunaga et al., 2015

Licensee PAGEPress, Italy

Pediatric Reports 2015; 7:5795

doi:10.4081/pr.2015.5795

rotation. Midgut volvulus was observed in 57 cases $(61.3 \%)$, and intestinal ischemia was observed in 23 (24.7\%). The standard procedure was performed in 86 cases (92.5\%), and enterectomy for necrotic intestine or enterostomy was performed in the remaining eight. Eighty-seven cases survived to discharge. The average length of follow-up after surgery was 7.7 years (median: 6.0 years).

Among the 87 cases that survived to discharge, postoperative intestinal obstruction was observed at least once in 22 cases (25.3\%), and three or more times in 12 cases. Twelve cases needed further surgery such as adhesiolysis or enterectomy (Table 2). The timing of the appearance of intestinal obstruction is shown in Figure 1. Most cases of first intestinal obstruction developed within two years following the Ladd procedure, though a few cases developed in the late period, after more than 10 years had passed.

Among the cases with intestinal obstruction, $13(59.0 \%)$ showed intestinal ischemia at the initial operation; this incidence was significantly high, although the rate in cases with associated surgical digestive disease was quite low (Table 3 ). Other clinical factors, including age at operation, weight at operation, classification, midgut volvulus, and procedure type were not significant risk factors for postoperative intestinal obstruction. All cases of intestinal obstruction that underwent reoperation were caused not by recurrent volvulus, but by 
adhesion or narrowing. Most of the induced adhesions were located between the intestine and the mesentery, and none was related to the wound.

\section{Discussion}

Previous studies have reported that the incidence of intestinal obstruction after the Ladd procedure is $6.1-23.9 \% \%^{3,7-9}$ In the present series, the incidence of postoperative intestinal obstruction that required hospitalization was $25.3 \%$, and surgical synechiotomy was required in $14.9 \%$ of patients. In other studies on postoperative intestinal obstructions after surgery for neonatal surgical digestive tract disease, the incidence of synechiotomy was reported as $2.3 \%$ in duodenal atresia, $108.0 \%$ in congenital diaphragmatic hernia, ${ }^{11}$ and $7.8 \%$ in gastroschisis. ${ }^{12}$ Compared with these values, the incidence of intestinal obstruction after the Ladd procedure is high.

Intestinal obstruction after a Ladd procedure results from recurrent midgut volvulus and adhesive bands. There were no cases of recurrent midgut volvulus among the 57 Ladd procedure patients reported by Murphy et al. or among the 87 cases reported by Ford et al:;,13 one case was observed among 161 reported by El-Gohary et al., ${ }^{3}$ and there have occasionally been case reports on the subject.4,5 Recurrent midgut volvulus as a complication after the Ladd procedure is not common but possible. Several decades ago, fearing that recurrent midgut volvulus might be caused by the narrowing of the mesenteric base due to the readhesion of the opened mesentery, Bill et al. reported a stabilization method that consisted of suturing the duodenum to the peritoneum over the right kidney and the cecum to the descending colon. ${ }^{14}$ In addition, they later reported a modified procedure that involved suturing the cecum to the left abdominal sidewall. ${ }^{15}$ Over time, however, it became clear that this intestinal stabilization procedure resulted in no improvement in the incidence of recurrent volvulus, ${ }^{13,16}$ and intestinal stabilization is hardly ever performed today. ${ }^{17}$ We also considered that intestinal fixing is not required to prevent recurrent volvulus after the Ladd procedure, but it is important to achieve adequate widening of the mesenteric base using the proper technique. There were, in fact, no cases of recurrent volvulus in the present series. On the other hand, few reports have focused on adhesive intestinal obstruction after the Ladd procedure. The present study demonstrated that the presence of intestinal ischemia at the first operation was the only risk factor for postoperative intestinal obstruction. In fact, 13 (59.1\%) of 22 cases with intestinal ischemia observed during the Ladd procedure developed postoperative adhesive intestinal obstruction. In contrast, neither the clinical entity nor midgut volvulus itself was a risk factor. When intestinal ischemia was not present, the incidence of postoperative adhesive intestinal obstruction was not increased, even in cases with midgut volvulus. In addition, contrary to our expectations, the presence of associated digestive surgical anomalies such as congenital diaphragmatic hernia or gastroschisis did not increase the incidence of intestinal obstruction. The intraoperative findings of 12 cases that had undergone synechiotomy but had not thus been cured revealed that most

Table 1. Patients characteristics (n=93).

\begin{tabular}{|c|c|}
\hline Characteristic & N. \\
\hline Male/female & $67 / 26$ \\
\hline $\begin{array}{l}\text { Associated digestive surgical anomalies (\%)* } \\
\text { Congenital diaphragmatic hernia } \\
\text { Duodenal atresia } \\
\text { Small intestinal atresia/stenosis } \\
\text { Gastroschisis } \\
\text { Omphalocele } \\
\text { Anorectal malformation } \\
\text { Others }\end{array}$ & $\begin{array}{c}28(30.1) \\
7 \\
6 \\
6 \\
3 \\
3 \\
2 \\
4\end{array}$ \\
\hline $\begin{array}{l}\text { Age at operation (\%) } \\
\text { Neonatal period } \\
\text { Infant period } \\
\text { Early childhood } \\
\text { Elementary school years }\end{array}$ & $\begin{aligned} 70 & (75.3) \\
7 & (7.5) \\
6 & (6.5) \\
10 & (10.8)\end{aligned}$ \\
\hline Weight at operation $(\mathrm{kg})^{\circ}$ & $5.2 \pm 7.0($ range $1.7-31.5)$ \\
\hline $\begin{array}{l}\text { Classification (\%) } \\
\text { Nonrotation } \\
\text { Incomplete rotation } \\
\text { Incomplete rotation with paraduodenal hernia }\end{array}$ & $\begin{array}{c}43(46.2) \\
48(51.6) \\
2(2.2)\end{array}$ \\
\hline Midgut volvulus (\%) & $57(61.3)$ \\
\hline Intestinal ischemia (\%) & $23(24.7)$ \\
\hline $\begin{array}{l}\text { Operative procedure (\%) } \\
\text { Standard Ladd procedure } \\
\text { Enterectomy } \\
\text { Enterostomy }\end{array}$ & $\begin{array}{c}86(92.5) \\
4(4.3) \\
4(4.3)\end{array}$ \\
\hline Survival to discharge (\%) & $87(93.5)$ \\
\hline Follow-up (years) ${ }^{\circ}$ & $7.7 \pm 7.4$ (range $0-28)$ \\
\hline
\end{tabular}

Table 2. Intraoperative findings at reoperation.

\begin{tabular}{lccc} 
Case Timing of the operation* & Cause of the obstruction & Operative procedure \\
1 & $11 \mathrm{mo}$ & Adhesion (ileum-mesentery) & Adhesiolysis \\
2 & $1 \mathrm{y}$ & Adhesion (ileum-mesentery) & Adhesiolysis \\
\hline 3 & $5 \mathrm{y}$ & Adhesion (ileum-mesentery) & Adhesiolysis \\
4 & $6 \mathrm{y}$ & Adhesion (ileum-mesentery) & Adhesiolysis \\
\hline 5 & $2 \mathrm{y}$ & Adhesion (jejunum-mesentery) & Enterectomy \\
6 & $6 \mathrm{y}$ & Adhesion (jejunum-mesentery) & Enterectomy \\
\hline 7 & $1 \mathrm{mo}$ & Adhesion (ileum-ileum) & Adhesiolysis \\
8 & $3 \mathrm{y}$ & Adhesion (ileum-ileum) & Adhesiolysis \\
\hline 9 & $9 \mathrm{mo}$ & Adhesion (duodenum-colon-omentum) & Adhesiolysis \\
10 & $1 \mathrm{y}$ & Narrowing of the mesoileum & Adhesiolysis \\
\hline 11 & $23 \mathrm{y}$ & Compression (dilated ileum to the ileocecal area) & Adhesiolysis + STEP \\
12 & $6 \mathrm{mo}$ & Adhesion (ileocecal area-mesentery) & Enterectomy \\
\hline *Postoperative years.mo, months y years STEP, serial transverse enteroplasty &
\end{tabular}

induced adhesions were located between the intestine and the mesentery, and none was related to the wound. These facts indicate that intestinal ischemia due to midgut volvulus causes serosal damage, and that adhesion area of the widening mesentery or other nearby intestinal serosa occurs during the process of healing. After the Ladd procedure, serosal damage associated with intestinal ischemia and the existence of a broad bare area of the mesentery were the most significant factors increasing the risk of postoperative intestinal obstruction. It is not evident that the associabetween intestinal serosa and the broad bare

\section{Table 1. Patients characteristics (n=93).}


tion between ischemic change and postoperative bowel obstruction is specific for malrotation, because there are few reports of the longterm complications following other diseases with intestinal ischemia, such as the internal hernia that resulted in postoperative bowel obstruction. However, it has been postulated that an early event in the pathogenesis of postoperative intestinal obstruction is the release of proinflammatory mediators, due to peritoneal breach and bowel handling. 18 There is no doubt that ischemic change leads to inflammatory cell activation and the start of the inflammatory cascade, so intestinal ischemia regardless of the underlying etiology can cause the peritoneal adhesion.

To prevent adhesive intestinal obstruction after the Ladd procedure, it is important to make the best possible effort to avoid intestinal ischemia, as well as intestinal adhesion to the broad bare area of the mesentery by widening of the mesenteric base. Since the efficacy of Seprafilm for preventing adhesive intestinal obstruction was reported in 1996,19 it has been commonly used in various clinical fields. ${ }^{20,21}$ Inoue et al. reported that, of five patients who underwent the Ladd procedure with Seprafilm, none developed a postoperative adhesive intestinal obstruction in the 24 months after surgery.22 Accordingly, we are considering applying Seprafilm to the manipulated mesentery as an effective preventive measure. On the other hand, it has been suggested that moderate adhesion in the peritoneal cavity plays some role in the prevention of recurrent volvulus, $2,23,24$ and that the use of an adhesive barrier film could therefore increase the risk of recurrent volvulus. However, if the use of Seprafilm can prevent adhesion between the widened mesenteries, re-narrowing of the mesenteric base can also be prevented, and the basic form of the Ladd procedure can be retained.

In recent years, reports on the Ladd procedure have more often dealt with the laparoscopic version of the surgery. 25 Although the

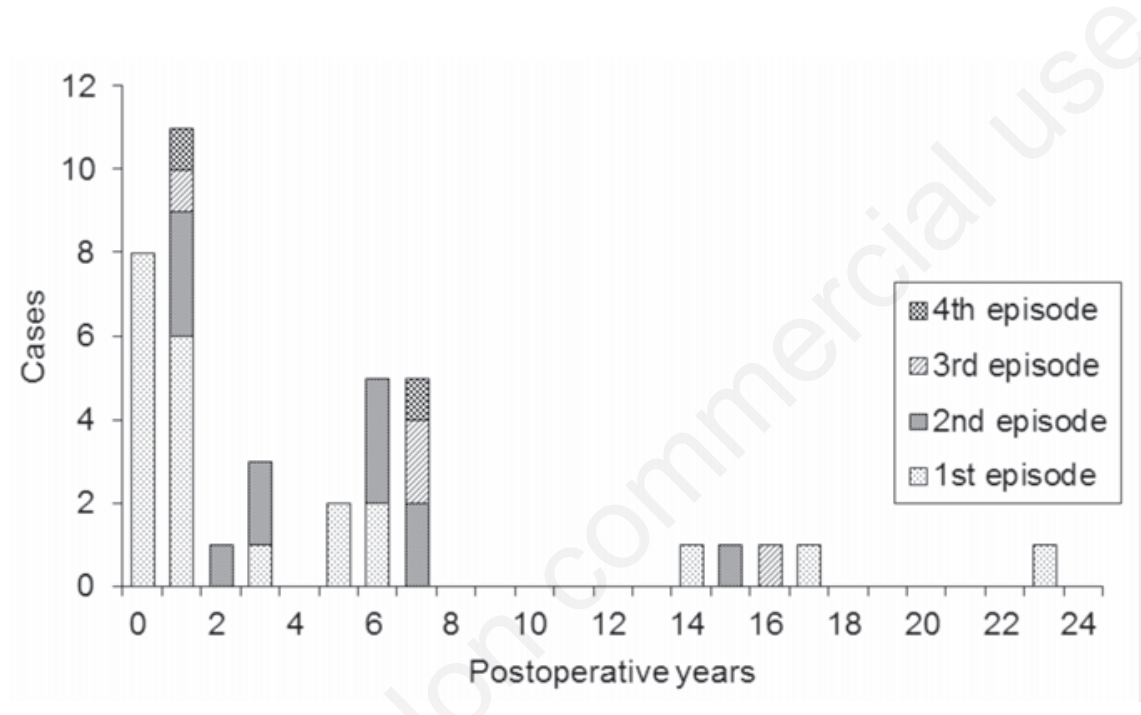

Figure 1. Timing of the appearance of postoperative intestinal obstruction.

Table 3. Correlations between clinical factors and postoperative intestinal obstruction.

\begin{tabular}{lccc} 
Clinical factors & $\begin{array}{c}\text { Postoperative intestinal obstruction } \\
\text { No } \\
(\mathrm{n}=65)\end{array}$ & $\begin{array}{c}\text { Yes } \\
(\mathrm{n}=22)\end{array}$ & P-value \\
\hline Age at operation (days)* & $570 \pm 395$ & $211 \pm 538$ & 0.243 \\
Weight at operation (kg)* & $5.6 \pm 7.9$ & $4.0 \pm 3.7$ & 0.497 \\
\hline Associated digestive surgical anomalies (\%) & $20(30.1)$ & $3(13.6)$ & 0.166 \\
Classification & 33 & 10 & 0.991 \\
$\quad$ Nonrotation & 38 & 12 & \\
\multicolumn{1}{l}{ Incomplete rotation } & $38(58.5)$ & $17(77.3)$ & 0.227 \\
Midgut volvulus (\%) & $9(13.8)$ & $13(59.1)$ & $<0.001^{\mathrm{a}}$ \\
\hline Intestinal ischemia (\%) & $61(93.8)$ & $20(90.9)$ & 0.650 \\
\hline Standard procedure (\%) & & &
\end{tabular}

* Data are presented as number or means \pm standard deviation. aStatistically significant.

laparoscopic Ladd procedure offers a cosmetic advantage and is also a useful procedure, there is considerable doubt over whether this procedure reduces the incidence of postoperative adhesive intestinal obstruction. In the present series, on the other hand, there were no cases in which adhesion related to the wound became the cause of an intestinal obstruction. In addition, inadequate mesenteric dissection with a persistently narrowed midgut mesentery under the restricted view can result in recurrent volvulus. Further investigation and more experience are needed.

\section{Conclusions}

Intestinal fixing is not required to prevent recurrent volvulus after the Ladd procedure, but it is important to achieve adequate widening of the mesenteric base using the proper technique. The risk of bowel obstruction after the Ladd procedure, on the other hand, is high. Moreover, patients with intestinal ischemia have an increased risk of bowel obstruction. This is thought to be caused by ischemic damage to the intestinal serosa and by peritoneum defects due to the widening of the mesentery. We are considering applying an adhesion barrier film to the manipulated mesentery as a preventive measure.

\section{References}

1. Ladd WE. Surgical diseases of the alimentary tract in infants. N Engl J Med 1936; 215:705-8.

2. Stockmann PT. Malrotation. In: Oldham KT, Colombani PM, Foglia RP, Skinner MA, eds. Principles and practice of pediatric surgery. Philadelphia: Lippincott Williams \& Wilkins; 2005. pp 1283-1296.

3. El-Gohary Y, Alagtal M, Gillick J. Long-term complications following operative intervention for intestinal malrotation: a 10 year review. Pediatr Surg Int 2010;26:2036.

4. Tanaka K, Takeda N, Takayasu H, et al. A case of recurrent midgut volvulus needing massive bowel resection 17 years after Ladd procedure. Jpn J Pediatric Surg 2012:48:76-80.

5. Biko DM, Anupindi SA, Hanhan SB, et al. Assessment of recurrent abdominal symptoms after Ladd procedure: clinical and radiographic correlation. J Pediatr Surg 2011;46:1720-5.

6. Touloukian RJ, Smith EI. Disorder of rotation and fixation. In: 0'Neill JA Jr, Rowe MI, Grosfeld JL, et al, eds. Pediatric Surgery. 5th ed. St Louis: Mosby-Year 
Book; 1998. pp 1199-1214.

7. Murphy FL, Sparnon AL. Long-term complications following intestinal malrotation and the Ladd's procedure: a 15 year review. Pediatr Surg Int 2006;22:326-9.

8. Mehall JR, Chandler JC, Mehall RL, et al. Management of typical and atypical intestinal malrotation. J Pediatr Surg 2002;37: 1169-72.

9. Feitz R, Vos A. Malrotation: the postoperative period. J Pediatr Surg 1997;32:1322-4.

10. Escobar MA, Ladd AP, Grosfeld JL, et al. Duodenal atresia and stenosis: long-term follow-up over 30 years. J Pediatr Surg 2004;39:867-71.

11. Jancelewicz T, Chiang M, Oliveira C, Chiu PP. Late surgical outcomes among congenital diaphragmatic hernia (CDH) patients: why long-term follow-up with surgeons is recommended. J Pediatr Surg 2013;48:93541.

12. Lepigeon K, Van Mieghem T, Vasseur Maurer S, et al. Gastroschisis - what should be told to parents? Prenat Diagn 2014;34:1-11.

13. Ford EG, Senac MO Jr, Srikanth MS, Weitzman JJ. Malrotation of the intestine in children. Ann Surg 1992;215:172-8.

14. Bill AH, Grauman D. Rationale and technic for stabilization of the mesentery in case of nonrotation of the midgut. J Pediatr Surg 1966;1:127-36.

15. Brennom WS, Bill AH. Prophylactic fixation of the intestine for midgut nonrotation. Surg Gynecol Obstet 1974;138:181.

16. Andrassy RJ, Mahour GH. Malrotation of the midgut in infants and children: a 25year review. Arch Surg 1981;116:158-60.

17. Stauffer UG, Herrmann P. Comparison of late results in patients with corrected intestinal malrotation with and without fixation of the mesentery. J Pediatr Surg 1980;15:9-12.

18. Vather R, O'Grady G, Bissett IP, Dinning PG. Postoperative ileus: mechanisms and future directions for research. Clin Exp Pharmacol Physiol 2014;41:358-70.

19. Becker JM, Dayton MT, Fazio VW, et al. Prevention of postoperative abdominal adhesions by a sodium hyaluronate-based bioresorbable membrane: a prospective, randomized, double-blind multicenter study. J Am Coll Surg 1996;183:297-306.

20. Vrijland WW, Tseng LN, Eijkman HJ, et al.
Fewer intraperitoneal adhesions with use of hyaluronic acid-carboxymethylcellulose membrane: a randomized clinical trial. Ann Surg 2002;235:193-9.

21. van der Wal JB, Iordens GI, Vrijland WW, et al. Adhesion prevention during laparotomy; long-term follow-up of a randomized clinical trial. Ann Surg 2011;253:1118-21.

22. Inoue M, Uchida K, Otake K, et al. Efficacy of Seprafilm for preventing adhesive bowel obstruction and cost-benefit analysis in pediatric patients undergoing laparotomy. J Pediatr Surg 2013;48:1528-34.

23. Mazeh H, Kaliner E, Udassin R. Three recurrent episodes of malrotation in an infant. J Pediatr Surg 2007;42:E1-3.

24. Panghaal V, Levin TL, Han B. Recurrent midgut volvulus following a Ladd procedure. Pediatr Radiol 2008;38:471-2.

25. Draus JM Jr, Foley DS, Bond SJ. Laparoscopic Ladd procedure: a minimally invasive approach to malrotation without midgut volvulus. Am Surg 2007;73:693-6. 